\title{
Mesocorticolimbic Pathways Encode Cue-Based Expectancy Effects on Pain
}

\author{
-YYiheng Tu, ${ }^{1}$ Yanzhi Bi, ${ }^{2,3}$ Libo Zhang, ${ }^{2,3}$ Hua Wei, ${ }^{2,3}$ and ${ }^{\circ} \mathrm{Li} \mathrm{Hu}{ }^{2,3}$ \\ ${ }^{1}$ Department of Psychiatry, Massachusetts General Hospital and Harvard Medical School, Charlestown, Massachusetts 02129, ${ }^{2}$ CAS Key Laboratory of \\ Mental Health, Institute of Psychology, Chinese Academy of Sciences, Beijing, China, 100101, and ${ }^{3}$ Department of Psychology, University of Chinese \\ Academy of Sciences, Beijing, China, 100101
}

\begin{abstract}
Expectation interacting with nociceptive input can shape the perception of pain. It has been suggested that reward-related expectations are associated with the activation of the ventral tegmental area (VTA), which projects to the striatum (e.g., nucleus accumbens [NAc]) and prefrontal cortex (e.g., rostral anterior cingulate cortex [rACC]). However, the role of these projection pathways in encoding expectancy effects on pain remains unclear. In this study, we leveraged a visual cue conditioning paradigm with a long pain anticipation period and collected magnetic resonance imaging (MRI) data from 30 healthy human subjects (14 females). At the within-subject level, whole-brain functional connectivity (FC) analyses showed that the mesocortical pathway (VTA-rACC FC) and the mesolimbic pathway (VTA-NAc FC) were enhanced with positive expectation but inhibited with negative expectation during pain anticipation period. Mediation analyses revealed that cue-based expectancy effects on pain were mainly mediated by the VTA-NAc FC, and structural equation modeling showed that VTA-based FC influenced pain perception by modulating pain-evoked brain responses. At the between-subject level, multivariate pattern analyses demonstrated that gray matter volumes in the VTA, NAc, and rACC were able to predict the magnitudes of conditioned pain responses associated with positive and/or negative expectations across subjects. Our results therefore advance the current understanding of how the reward system is linked to the interaction between expectation and pain. Furthermore, they provide precise functional and structural information on mesocorticolimibic pathways that encode within-subject and between-subject variability of expectancy effects on pain.
\end{abstract}

Key words: conditioned pain responses; expectancy effects; mesocortical pathway; mesolimbic pathway; ventral tegmental area (VTA)

\section{Significance Statement}

Studies have suggested that reward-related expectation is associated with the activation of the VTA, which projects to the striatum and prefrontal cortex. However, the role of these projection pathways in encoding expectancy effects on pain remains unclear. Using multimodality MRI and a visual cue conditioning paradigm, we found that the functional connectivity and gray matter volumes in key regions (the VTA, NAc, and rostral ACC) within the mesocorticolimbic pathways encoded expectancy effects on pain. Our results advance the current understanding of how the reward system is linked to the interaction between expectation and pain, and provide precise functional and structural information on mesocorticolimbic pathways that encode expectancy effects on pain.

\section{Introduction}

Pain is a highly subjective experience that can be influenced by a variety of psychological factors (Wiech et al., 2008). One clear

\footnotetext{
Received May 11, 2019; revised 0ct. 25, 2019; accepted 0ct. 31, 2019.

Author contributions:Y.T. and L.H. designed research;Y.T. and H.W. performed research; Y.T. analyzed data; Y.T. and L.H. wrote the paper; Y.B., L.Z., and L.H. edited the paper.

This work was supported by the National Natural Science Foundation of China 31671141 and 31822025, the 13th Five-year Informatization Plan of Chinese Academy of Sciences XXH13506, and the Scientific Foundation project of Institute of Psychology, Chinese Academy of Sciences Y6CX021008. We thank Dr. Falk Eippert (Max Planck Institute for Human Cognitive and Brain Sciences) for valuable comments and suggestions in data analyses and manuscript preparation.

The authors declare no competing financial interests.

Correspondence should be addressed to Li Hu at huli@psych.ac.cn.
}

example is that expectation of nociceptive input can modulate the perception of pain (Atlas and Wager, 2012; Fields, 2018). To investigate the relationship between expectation and pain, studies invoke conditioning to establish a link between a cue and increased or reduced pain that follows, thus creating predictive knowledge (i.e., expectation) that modulates future pain responses to the same cue (Wager et al., 2004; Jensen et al., 2012). Although the neural mechanisms underlying the interaction between expectation and pain are still under investigation, neuro-

https://doi.org/10.1523/JNEUROSCI.1082-19.2019

Copyright $\odot 2020$ the authors 
imaging studies using functional magnetic resonance imaging (fMRI) and positron emission tomography have offered insights into how predictive cues are processed by the coordination of complex brain circuits (e.g., brainstem, higher-order cortical and subcortical brain regions), and then modulate pain and painrelated brain responses (Wager et al., 2004, 2007; Koyama et al., 2005; Seymour et al., 2005; Atlas et al., 2010; Kong et al., 2013; Shih et al., 2019).

Among several neurobiological models proposed for explaining the relationship between expectation and pain-related behaviors (Enck et al., 2008; Büchel et al., 2014), brain reward circuity has been widely studied in both basic and clinical settings. It has been suggested that expectation is closely related to the activation of tegmental or prefrontal dopaminergic neurons, which project to the dorsal and ventral striatum (de la Fuente-Fernández et al., 2001, 2002; Lidstone et al., 2010), and may affect the circuitry of the basal ganglia (Benedetti et al., 2004). In particular, the mesocorticolimbic pathways, which originate from the ventral tegmental area (VTA) and project primarily to the frontal lobe (e.g., rostral anterior cingulate cortex $[\mathrm{rACC}]$ ) and ventral striatum (e.g., nucleus accumbens [NAc]) (Gardner and Ashby, 2000), form part of the key circuits that are typically implicated in reward and motivation behaviors (Enck et al., 2008; Scott et al., 2008; Schweinhardt et al., 2009).

While previous studies have focused on investigating rewardrelated brain responses in the frontal lobe and striatum, it is unlikely that isolated brain regions would be sufficient to achieve relevant functions (e.g., reward, aversion). The role of mesocorticolimbic pathways (e.g., from VTA to rACC and from VTA to NAc) in mediating cue-based expectancy effects on pain remains elusive, which is the primary aim of the present study. Moreover, evidence from fMRI and structural MRI has suggested that a more efficient reward system, for example, larger NAc responses to reward cues (Scott et al., 2007) and higher gray matter densities of NAc and PFC (Schweinhardt et al., 2009), may be associated with stronger reward-related behaviors. Therefore, we also aimed to investigate whether the functional and structural properties of mesocorticolimbic pathways can explain individual differences of conditioned pain responses.

To answer these questions, we leveraged a visual cue conditioning paradigm with a long pain anticipation period ( $15 \mathrm{~s})$ for each trial and collected structural MRI and fMRI data from 30 healthy participants. At the within-subject level, we performed a VTA-based whole-brain functional connectivity (FC) analysis to identify pathways involved in cue-based pain modulation. In addition, we conducted a mediation analysis to assess the potential mediatory effect of anticipatory VTA-based FC on the relationship between expectation and pain perception. We applied structural equation modeling to explore whether VTA-based FC influences pain perception by modulating pain-evoked brain responses. At the between-subject level, we applied a multivariate pattern analysis (MVPA) to assess the possibility of functional and structural features (VTA-based FC and gray matter volume [GMV], respectively) associated with mesocorticolimbic pathways to predict conditioned pain responses.

\section{Materials and Methods}

\section{Subjects}

Thirty healthy participants without any psychiatric or neurologic disorders were enrolled in this study. One subject was excluded from data analysis due to an incomplete MRI scan. The final sample consisted of 29 participants ( 14 females; age $22.0 \pm 1.9$ years). To detect the effectiveness of sample size for exploring the cue-based expectancy effects on pain, we performed a power analysis for $F$ tests using $G^{*}$ power (a free online software for power analysis; available at http://www.gpower.hhu.de/ en.html) and set statistical power at 0.8 with a medium effect size $(f=$ 0.25 ) and significance level at 0.05 (Cunningham and McCrum-Gardner, 2007). The result showed a minimum sample size of 28 for detecting main effects. As for the power to determine the effects of conditioned expectation on brain responses, the sample size of our study was determined based on the sample sizes of previous studies that similarly aimed to investigate expectancy-based pain modulation using fMRI techniques (Wager et al., 2004, 2011; Atlas et al., 2010; Jensen et al., 2015). All protocols were approved by the Institutional Review Board of Southwest University, and all participants provided written and signed informed consent.

\section{Experimental procedures}

The experiment consisted of three phases: calibration phase, conditioning phase, and test phase. Calibration and conditioning phases were performed $24 \mathrm{~h}$ before the test phase.

Calibration phase. Nociceptive somatosensory stimuli (intraepidermal electrical stimuli) were delivered using an electrical stimulator (model DS7A; Digitimer) with three stainless-steel concentric bipolar needle electrodes separated by an equal distance of $6 \mathrm{~mm}$. Each electrode consisted of a needle cathode (length: $0.1 \mathrm{~mm}$; $: 0.2 \mathrm{~mm}$ ) surrounded by a cylindrical anode $(\varnothing: 1.4 \mathrm{~mm})$. Each stimulus consisted of 6 rapidly succeeding constant-current, square-wave pulses at $60 \mathrm{~Hz}(0.5 \mathrm{~ms}$ duration for each pulse; $100 \mathrm{~ms}$ for the whole stimulus). The electrodes were placed over the left volar forearm. For each subject, the ascending method of limits (pulses with an ascending current starting from $0.2 \mathrm{~mA}$ and increasing in steps of $0.1 \mathrm{~mA}$ ) were applied in the calibration phase to determine the stimulus intensity that would represent "low pain" ( 2 on a $0-10$ numerical rating scale [NRS]; 0: no pain; 10: worst pain that subject can tolerate), "moderate pain" (4 on the NRS), and "high pain" (6 on the NRS). Subjects were instructed to move a slider to rate the intensity of pain perception. This procedure was repeated three times for each subject, and the averaged stimulus intensities were calculated for the following experiment. In addition, the averaged stimulus intensities were presented several times in random orders to ensure rating consistency. Intraepidermal electrical stimuli were shown to preferentially activate the $\mathrm{A} \delta$ nociceptive fibers, and the simultaneous activation of the $\mathrm{A} \beta$ fibers cannot be completely ruled out considering that a wide range of stimulus intensities were used (Mouraux et al., 2010).

Conditioning phase. After the calibration phase, participants were given the following instruction: "You are about to see some pictures on the screen. Each picture is paired with a pain stimulus on your arm. Your task is to focus on the screen at all time, and after each pain stimulus, you are required to rate how much pain you felt on your arm using the same $0-10$ NRS as in the calibration phase."

A $60 \mathrm{~Hz}, 19$-inch Dell monitor was used for visual presentations, and the screen resolution was $1024 \times 768$ pixels. The experiment was programmed in E-Prime 3.0 software (Psychology Software Tools). In each trial, a visual cue presented on the screen was followed by a pain stimulus $15 \mathrm{~s}$ later (from the offset of visual cue to the onset of pain stimulus). Subjects were required to rate the intensity of pain perception on the same $0-10$ NRS. In the conditioning phase, two types of cues (a white plus mark and a white minus mark) were presented 20 times each in a random order. The white plus mark cue was followed by a high pain stimulus, and the white minus mark cue by a low pain stimulus (Fig. $1 \mathrm{~A}$ ). The timings of a typical trial in the conditioning phase are displayed in Figure $1 C$. The intertrial interval ranged from 10 to $12 \mathrm{~s}$. To ensure that subjects remained attentive to external sensory stimuli, the conditioning phase was divided into two sessions ( $\sim 15 \mathrm{~min}$ each). Subjects had the opportunity to rest for $\sim 1$ min between sessions.

After the conditioning phase, all participants verbally confirmed that the stimuli following the white plus mark was more painful than the stimuli following the white minus mark using the same $0-10$ NRS.

Test phase. The test phase was performed during the MRI scan, and subjects had a response device in their right hand to rate the intensity of pain perception in the scanner. Before fMRI data collection, the stimulus intensity was calibrated again in the MRI scanner, and five low-pain trials 
and five high-pain trials were delivered to the subjects to familiarize them with the sensation.

Subjects were then instructed, "You are about to see the same pictures on the screen, and each picture will be paired with a pain stimulus on your arm, just like before. The only difference is that this time there will be a picture of a circle, which you have not been exposed to before. Your task is to focus on the screen at all time, and after each pain stimulus you are required to rate how much pain you felt on your arm using the same $0-10$ NRS."

In the test phase, all cues were followed by pain stimuli of the same intensity (i.e., moderate pain, 4 on the NRS) to test the conditioning effect (Fig. 1B). The timings of a typical trial in the test phase are detailed in Figure 1D. The intertrial interval was between 10 and $12 \mathrm{~s}$. The test phase was divided into three sessions of 15 trials (45 trials in total), each of which contained 5 trials for each of the 3 different cues (a white plus mark, white minus mark, and white circle mark). Subjects could rest for $1 \mathrm{~min}$ between consecutive sessions.

After the test phase, all participants confirmed that they expect to receive more painful stimuli following the white plus mark compared with the white minus mark.

\section{Behavioral data analysis}

The positive expectancy effect was defined as the difference between perceived intensity to moderate pain stimuli following the low cue (white minus mark) and following the neutral cue (white circle mark) in the test phase. The negative expectancy effect was defined as the difference between perceived intensity to moderate pain stimuli following the high cue (white plus mark) and following the neutral cue (white circle mark) in the test phase. Adding a neutral cue (i.e., white circle mark) enabled us to differentiate the positive expectancy effects (low cue vs neutral cue) from the negative expectancy effects (high cue vs neutral cue). The comparison of perceived pain intensities following different cues was assessed using a oneway repeated-measures ANOVA (see Fig. 2A). When the main effect was significant, post hoc paired-sample $t$ tests were performed and $p$ values were Bonferroni corrected.
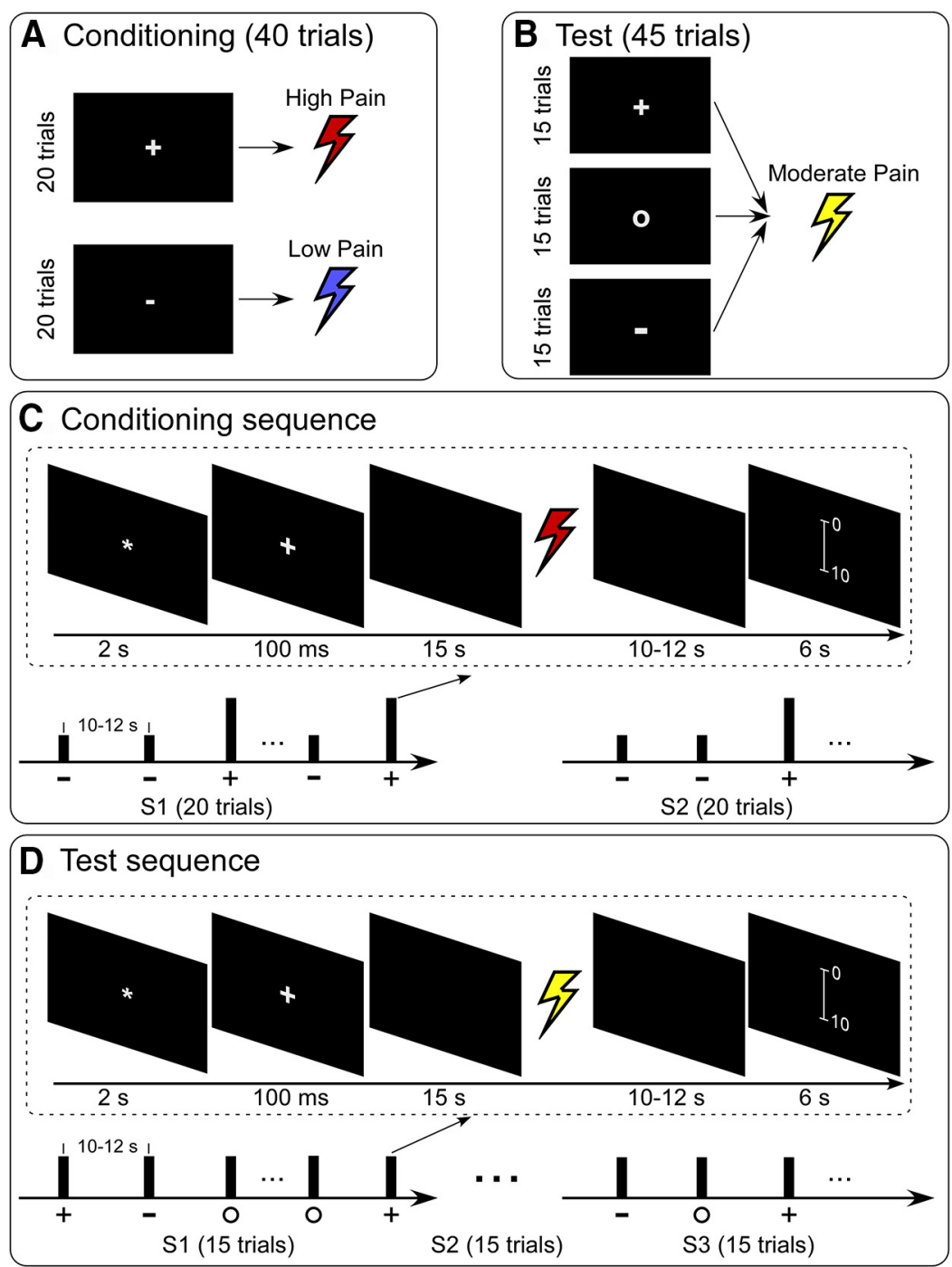

Figure 1. Experimental design. $\boldsymbol{A}$, In the conditioning phase, two types of cues (white plus mark and white minus mark) were presented 20 times each in a random order. The white plus mark cue was followed by a high pain stimulus, and the white minus mark cue by a low pain stimulus. $\boldsymbol{B}$, In the test phase, three types of cues (white plus mark, white circle mark, and white minus mark) were presented 15 times each in a random order. All cues were followed by stimuli of the same intensity (i.e., moderate pain). C, There were two sessions in the conditioning phase, and each session included 20 trials with two types of cues (white plus mark and white minus mark) displayed in random order. Subjects could rest for 1 min between each session. $\boldsymbol{D}$, There were three sessions in the test phase, and each session included 15 trials with three types of cues (white plus mark, white minus mark, and white circle mark) displayed in random order. Subjects could rest for 1 min between consecutive sessions.

\section{MRI data acquisition}

MRI data were acquired using a Siemens 3.0 Tesla Trio scanner with a standard 32-channel head coil at the Key Laboratory of Cognition and Personality (Ministry of Education) of Southwest University (China). Functional images were obtained using a gradient EPI sequence with the following parameters ( $\mathrm{TR}=1500 \mathrm{~ms}, \mathrm{TE}=29 \mathrm{~ms}, 25$ slices, slice thickness $=5.0 \mathrm{~mm}$, interslice gap $=0.5 \mathrm{~mm}$, in-plane resolution $=3 \mathrm{~mm} \times$ $3 \mathrm{~mm}, \mathrm{FOV}=192 \times 192 \mathrm{~mm}^{2}$, data matrix $=64 \times 64$, flip angle $=90^{\circ}$ ). A high-resolution, 3D T1-weighted structural image was acquired using a MPRAGE sequence $\left(1 \mathrm{~mm}^{3}\right.$ isotropic voxel, FOV $\left.=256 \times 256 \mathrm{~mm}^{2}\right)$ after functional imaging.

\section{fMRI data analysis}

fMRI data preprocessing. fMRI data were preprocessed using SPM12 (Wellcome Trust Center for Neuroimaging, London). The first five volumes were discarded to allow for signal equilibration. Images were slice timing corrected using the middle slice and realigned to the first scan. The resulting images were normalized to the MNI space (resampling voxel size $=3 \times 3 \times 3 \mathrm{~mm}^{3}$ ) (Ashburner and Friston, 2005). To mini- mize the effect of head motion in the following fMRI analyses, 6 motion estimates and 2 physiological time series (white matter and CSF) were regressed out of the normalized images.

Due to the small size of the VTA and its proximity to other brainstem structures, the brainstem, isolated using the SUIT toolbox (http:// www.diedrichsenlab.org/imaging/suit.htm), was not spatially smoothed, and the time course of the isolated VTA seed was extracted. The rest of the brain, excluding the brainstem, was smoothed with a $5 \mathrm{~mm}$ FWHM Gaussian smoothing kernel.

GLM analysis. Single-subject fMRI data were analyzed using a GLM approach, including regressors for three types of visual cues (low, neutral, and high cues) and pain stimuli. The BOLD signals were modeled as a series of events (visual cues and pain stimuli) using a stick function, and convolved with a canonical HRF. To identify brain responses to pain stimuli, group-level statistical analyses were performed using a random-effects analysis with a one-sample $t$ test, as implemented in SPM12. The significance threshold was set as $p<$ 0.001 at the voxel level and $p_{\mathrm{FDR}}<0.05$ at the cluster level (false 
A Pain ratings

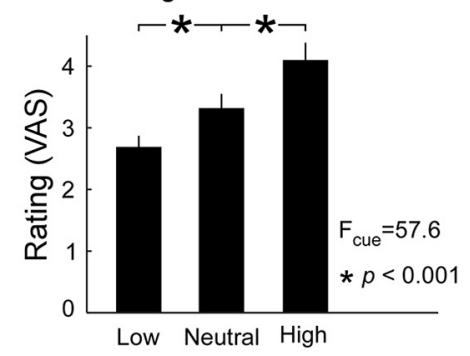

C Neutral vs. Low during stimulation

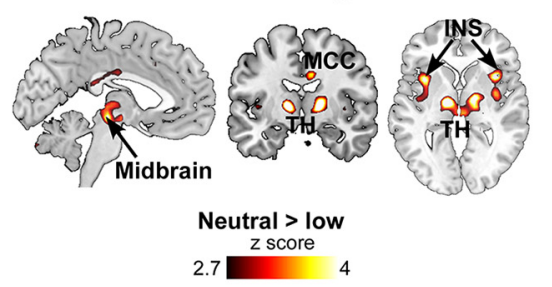

\section{E Pain evoked brain responses}
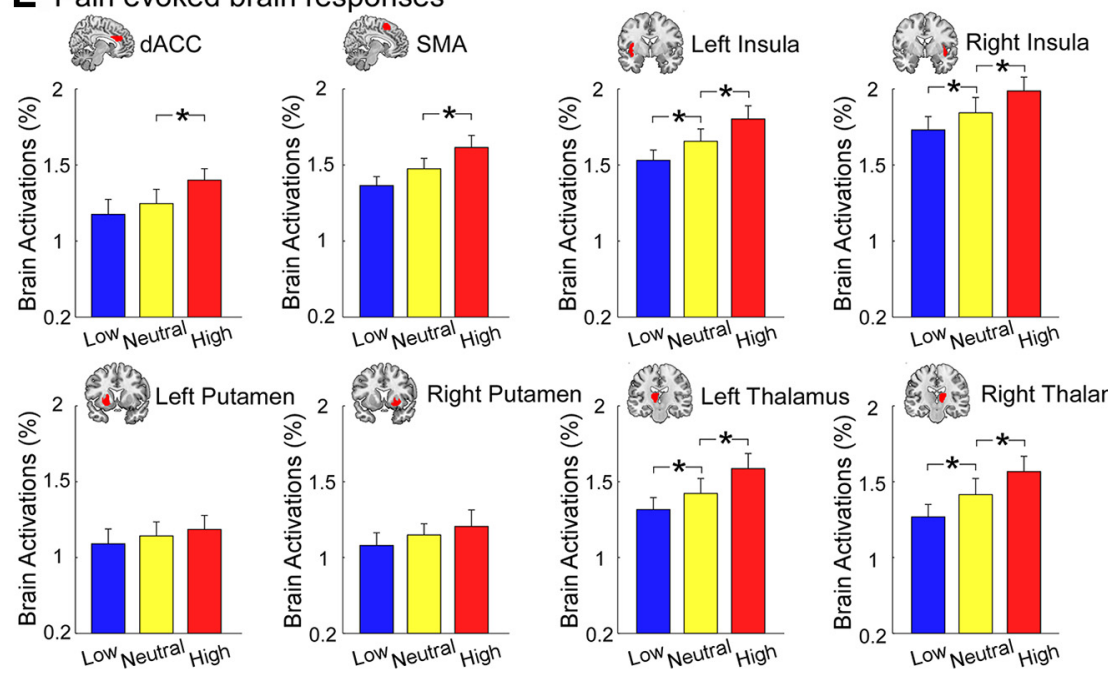

Figure 2. Pain and conditioned pain-related brain responses. $A$, Significant differences of perceived pain intensities following low, neutral, and high cues were observed across all subjects. B, Pain stimuli elicited brain activations in the dACC, SMA, putamen (PUT), caudate (CAU), thalamus (TH), and insula (INS). C, During the pain experience period, positive expectation induced decreased brain activations in the midbrain, MCC, INS, and TH. D, Negative expectation induced increased brain activations in the midbrain, dorsal lateral PFC (DLPFC), MCC, SMA, dACC, TH, and INS during the pain experience period. $\boldsymbol{B}-\boldsymbol{D}$, All results were thresholded at $p<0.001$ at voxel level and $p_{\mathrm{FDR}}<0.05$ at cluster level. $\boldsymbol{E}$, Pain-evoked brain responses at representative ROls. dACC, SMA, bilateral INS, bilateral PUT, and bilateral TH were selected as ROIs. We built these ROIs based on the conjunction of pain-responsive regions in $\boldsymbol{B}$ and the standard regions in Automated Anatomical Labeling atlas. Pain-evoked brain responses in these ROIs were extracted and compared between different cues (low, neutral, and high) using a one-way repeated-measures ANOVA and post hoc paired-sample $t$ tests when the main effect was significant. Error bars represent standard errors of mean. ${ }^{*} p<$ 0.05 (FDR correction).

discovery rate $[\mathrm{FDR}]$ correction for multiple comparisons) in the whole-brain exploratory analyses.

To assess conditioned expectancy effects, contrast images were computed for the low cue versus neutral cue and for the high cue versus neutral cue for anticipation and experience of pain separately, using a random-effects analysis with a paired-sample $t$ test in SPM12. Anticipation of pain was defined as the period between the presentation of visual cues and the delivery of pain stimuli, and experience of pain was defined as the period between the delivery of pain stimuli and the rating of pain perception. The significance threshold was set as $p<0.001$ at the voxel level and $p_{\mathrm{FDR}}<0.05$ at the cluster level in the whole-brain exploratory analyses.

VTA-based FC analyses. To investigate the role of mesocorticolimbic pathways in mediating conditioned expectancy effects, we selected the
VTA as the seed for the following seed-based FC analyses (see Fig. 4A). The probabilistic atlas of VTA was defined in an independent sample of 50 participants from a previous study (mean \pm SD volume: $440.98 \pm 100.6 \mathrm{~mm}^{3}$ ) (Murty et al., 2014) and thresholded to $75 \%$ (as suggested by Murty et al., 2017) in the present study. The VTA-based FC during the pain anticipation period was estimated using seed-based correlation analysis (Cisler et al., 2014; Cole et al., 2014). For each single trial, the averaged time series across all voxels of the VTA seed in the anticipation period ( $15 \mathrm{~s}$ in total; $10 \mathrm{scans}$ ) was extracted as the reference time series. Pearson's correlation analysis was performed to produce individual-level correlation maps of all voxels within the whole brain that represented their relationship with the reference time series of the VTA. Seed-based correlation analysis, rather than a psychophysiological interaction approach, was adopted in the present study, since previous studies have suggested that correlation analysis may be more suitable than psychophysiological interaction when assessing the differences in FC between experimental conditions (Di et al., 2018), especially for event-related fMRI data (Cisler et al., 2014).

The VTA-based whole-brain FC ( $r$ value) maps were Fisher's $z$-transformed to increase normality of the data for the following statistical analyses: (1) A one-sample $t$ test against 0 was performed to reveal brain regions that were significantly correlated with VTA during pain anticipation and averaged over all cues. The resulting statistical map was set at a threshold of $p<0.001$ at the voxel level and $p_{\mathrm{FDR}}<$ 0.05 at the cluster level in the whole-brain exploratory analyses. (2) Paired-sample $t$ tests were performed to show significant differences in positive expectation (low cue vs neutral cue) and negative expectation (high cue vs neutral cue) contrasts. The resulting statistical maps were set at a threshold of $p<0.001$ at the voxel level and $p_{\text {FDR }}<0.05$ at the cluster level (i.e., within the brain regions showing significant VTA-based FC; see Fig. 4A).

After identifying brain regions with significant differences in VTA-based FC in positive expectation and negative expectation contrasts, we calculated their overlaps (by finding the conjunct regions of Fig. $4 B, C$ ) for the following analysis (i.e., $\mathrm{rACC}_{\text {overlap }}$ and $\mathrm{NAc}_{\text {overlap; }}$ for details, see Results). Specifically, single-trial FC between VTA and rACC $_{\text {overlap }}$, as well as between

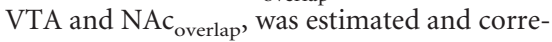
lated with the corresponding single-trial perceived pain intensities using Pearson's correlation analysis to investigate their relationship in the pain anticipation period. To minimize the influence of individual differences (Hu and Iannetti, 2019; Tu et al., 2019c), single-trial perceived pain intensities were normalized within each subject by subtracting their mean and dividing by their SD before performing the correlation analysis. The obtained correlation coefficients were Fisher's $z$-transformed, and the resulting $z$ values were compared against 0 using a one-sample $t$ test.

Control seed-based analyses. Due to the small size of the VTA and its adjacency to substantia nigra (SN), we performed control analyses with $\mathrm{SN}$ as the seed to demonstrate the robustness of results obtained from VTA-based analyses. The $\mathrm{SN}$ is the origin of the nigrostriatal pathway, 
Table 1. Clusters that exhibited significant brain activations and deactivations to pain stimuli ${ }^{a}$

\begin{tabular}{|c|c|c|c|c|c|c|c|c|c|}
\hline \multicolumn{5}{|c|}{ Activation clusters } & \multicolumn{5}{|c|}{ Deactivation clusters } \\
\hline Area & Side & Peak $x / y / z$ & $T$ & Size & Area & Side & Peak $x / y / z$ & $T$ & Size \\
\hline SMA & L & $-6,14,49$ & 8.5 & 283 & ParaHipp & L & $-30-34-17$ & -7.8 & 30 \\
\hline SMA & $\mathrm{R}$ & $8,20,39$ & 8.2 & 208 & ParaHipp & $\mathrm{R}$ & $27-34-17$ & -7.6 & 31 \\
\hline Insula & L & $-33,20,1$ & 7.5 & 361 & - & - & - & - & - \\
\hline Insula & $\mathrm{R}$ & $36,17,1$ & 7.7 & 323 & - & - & - & - & - \\
\hline Thalamus & $\mathrm{L}$ & $-9,-13,4$ & 7.4 & 164 & - & - & - & - & - \\
\hline Thalamus & $\mathrm{R}$ & $13,-13,4$ & 8.0 & 165 & - & - & - & - & - \\
\hline Caudate & $\mathrm{L}$ & $-11,11,4$ & 6.6 & 179 & - & - & - & - & - \\
\hline Caudate & $\mathrm{R}$ & $14,10,4$ & 6.7 & 168 & - & - & - & - & - \\
\hline Putamen & $\mathrm{L}$ & $-17,14,5$ & 6.4 & 119 & - & - & - & - & - \\
\hline Putamen & $\mathrm{R}$ & $17,7,-6$ & 6.6 & 128 & - & - & - & - & - \\
\hline $\mathrm{ACC}$ & L & $-6,34,26$ & 6.2 & 102 & - & - & - & - & - \\
\hline $\mathrm{ACC}$ & $\mathrm{R}$ & $10,30,27$ & 6.4 & 126 & - & - & - & - & - \\
\hline SI/SII & L & $-33,-58,43$ & 9.8 & 283 & - & - & - & - & - \\
\hline SI/SII & $\mathrm{R}$ & $60,-46,40$ & 10.0 & 426 & - & - & - & - & - \\
\hline Precuneus & L & $-12,-67,52$ & 8.7 & 53 & - & - & - & - & - \\
\hline Precuneus & $\mathrm{R}$ & $15,-67,40$ & 11.7 & 110 & - & - & - & - & - \\
\hline Cerebellum & L & $-6,-76,-20$ & 12.1 & 989 & - & - & - & - & - \\
\hline Cerebellum & $\mathrm{R}$ & $36,-58,-29$ & 10.5 & 397 & - & - & - & - & - \\
\hline
\end{tabular}

asI, Primary somatosensory cortex; SII, secondary somatosensory cortex; ParaHipp, parahippocampus.

Table 2. Clusters that exhibited significant differences between cues during pain experience ${ }^{a}$

\begin{tabular}{|c|c|c|c|c|c|c|c|c|c|}
\hline \multicolumn{5}{|l|}{ High > neutral } & \multicolumn{5}{|c|}{ Neutral > low } \\
\hline Area & Side & Peak x/y/z & $\mathrm{T}$ & Size & Area & Side & Peak $x / y / z$ & $\mathrm{~T}$ & Size \\
\hline Insula & L & $-37,5,-5$ & 6.8 & 300 & Insula & L & -36111 & 3.7 & 33 \\
\hline Insula & $\mathrm{R}$ & $39,11,-5$ & 6.9 & 320 & Insula & $\mathrm{R}$ & $3614-2$ & 4.0 & 36 \\
\hline $\mathrm{ACC}$ & $L, R$ & $-11,24,31$ & 3.7 & 290 & - & - & - & - & - \\
\hline SMA & $L, R$ & $6,17,46$ & 3.6 & 75 & - & - & - & - & - \\
\hline Putamen & L & $-31,6,-5$ & 6.1 & 54 & - & - & - & - & - \\
\hline Putamen & $\mathrm{R}$ & $30,10,-4$ & 6.2 & 116 & - & - & - & - & - \\
\hline Thalamus & $L$ & $-14,-6,0$ & 3.8 & 42 & Thalamus & L & $-15-137$ & 5.2 & 58 \\
\hline Thalamus & $\mathrm{R}$ & $16,-10,0$ & 3.9 & 92 & Thalamus & $\mathrm{R}$ & $15-107$ & 5.1 & 80 \\
\hline $\mathrm{MCC} / \mathrm{PCC}$ & $L, R$ & $9,-25,31$ & 5.6 & 257 & $\mathrm{MCC} / \mathrm{PCC}$ & $L, R$ & $9-2831$ & 3.6 & 43 \\
\hline DLPFC & $\mathrm{L}$ & $-32,51,6$ & 4.4 & 33 & - & - & 一 & - & - \\
\hline DLPFC & $\mathrm{R}$ & $41,50,4$ & 4.6 & 69 & - & - & - & - & - \\
\hline $\begin{array}{l}\text { Midbrain } \\
\text { High < neutral }\end{array}$ & $L, R$ & $09,-21,-5$ & 3.1 & 35 & $\begin{array}{l}\text { Midbrain } \\
\text { Low > ne }\end{array}$ & $L, R$ & $-5-22-9$ & 3.8 & 55 \\
\hline Area & Side & Peak x/y/z & $\mathrm{T}$ & Size & Area & Side & Peak x/y/z & $\mathrm{T}$ & Size \\
\hline Angular & $\mathrm{L}$ & $-45,-76,31$ & -4.0 & 30 & - & - & - & - & - \\
\hline Angular & $\mathrm{R}$ & $53,-68,28$ & -4.1 & 42 & - & - & - & - & - \\
\hline
\end{tabular}

${ }^{a}$ DLPFC, Dorsal lateral prefrontal cortex.

which transmits dopamine from $\mathrm{SN}$ to the dorsal striatum (e.g., the caudate, putamen). Different from mesocortical and mesolimbic pathways, the nigrostriatal pathway and the dorsal striatum are not usually associated with conditioned expectation on pain. The probabilistic atlas of SN was also defined in an independent sample of 50 participants from the previous study (Murty et al., 2014) (mean \pm SD volume: $972.4 \pm$ $267.5 \mathrm{~mm}^{3}$ ) and thresholded to $75 \%$. The SN-based FC analyses were identical to the VTA-based FC analyses, which have been detailed in the previous section. A high-resolution illustration of the VTA and SN seeds is provided in Fig. 4-1 (available at https://doi.org/10.1523/ JNEUROSCI.1082-19.2019.f4-1).

Mediation analyses. We performed bootstrapped mediation analyses to assess the mediatory role of anticipatory VTA-based FC (i.e., VTArACC and VTA-NAc) on the relationship between cue and pain perception. The PROCESS macro (version 2.16.3) in SPSS (IBM, version 22.0) was used with 1000 bootstrap samples, which identified 95\% CIs for model components. With categorical values as the independent variable (coded as 1, 0, and -1 for low, moderate, and high cues, respectively) (Hayes and Preacher, 2014) and perceived pain intensities as the outcome, we tested three different models: (1) VTA-NAc FC as a mediator, (2) VTA-rACC FC as a mediator, and (3) VTA-NAc and VTA-rACC FCs as two mediators. A significant mediation occurs when bootstrapped upper and lower 95\% CIs do not include 0 (Hayes and Preacher, 2014).
Structural equation modeling. To explore whether anticipatory VTAbased FC influences pain perception by modulating pain-evoked brain responses, structural equation modeling with maximum likelihood estimation was performed using Amos (IBM, version 22.0). Specifically, anticipatory VTA-based FC was the predictor and loaded by VTA-NAc FC and VTA-rACC FC. The outcome was perceived pain intensity. Painevoked brain responses were the mediator and loaded by BOLD responses in the bilateral thalamus and bilateral insula. These two brain regions were selected because (1) they were significantly activated by pain stimuli (see Fig. 2B), (2) pain-evoked brain responses were significantly stronger following neutral cues than low cues (see Fig. 2C), and (3) pain-evoked brain responses were significantly stronger following high cues than neutral cues (see Fig. 2D). The ROIs of the thalamus and insula were defined as the overlapping portions of the thresholded statistical maps in Figure $2 B-D$, and $\beta$ estimates at the individual level, extracted from the two ROIs following different cues, were used as the BOLD responses.

The model fit was assessed using the following criteria: ratio of $\chi^{2}$ to degrees of freedom $\left(\chi^{2} / \mathrm{df}\right)<2$ (Kline, 2015), root mean square error of approximation $\leq 0.06$ (Hu and Bentler, 1999), both goodness-of-fit index and adjusted goodness-of-fit index $\geq 0.90$, and both comparative fit index and normed fit index $\geq 0.95$ (Hooper et al., 2008). To assess the significance of the indirect and direct effects, bias-corrected 95\% CIs 


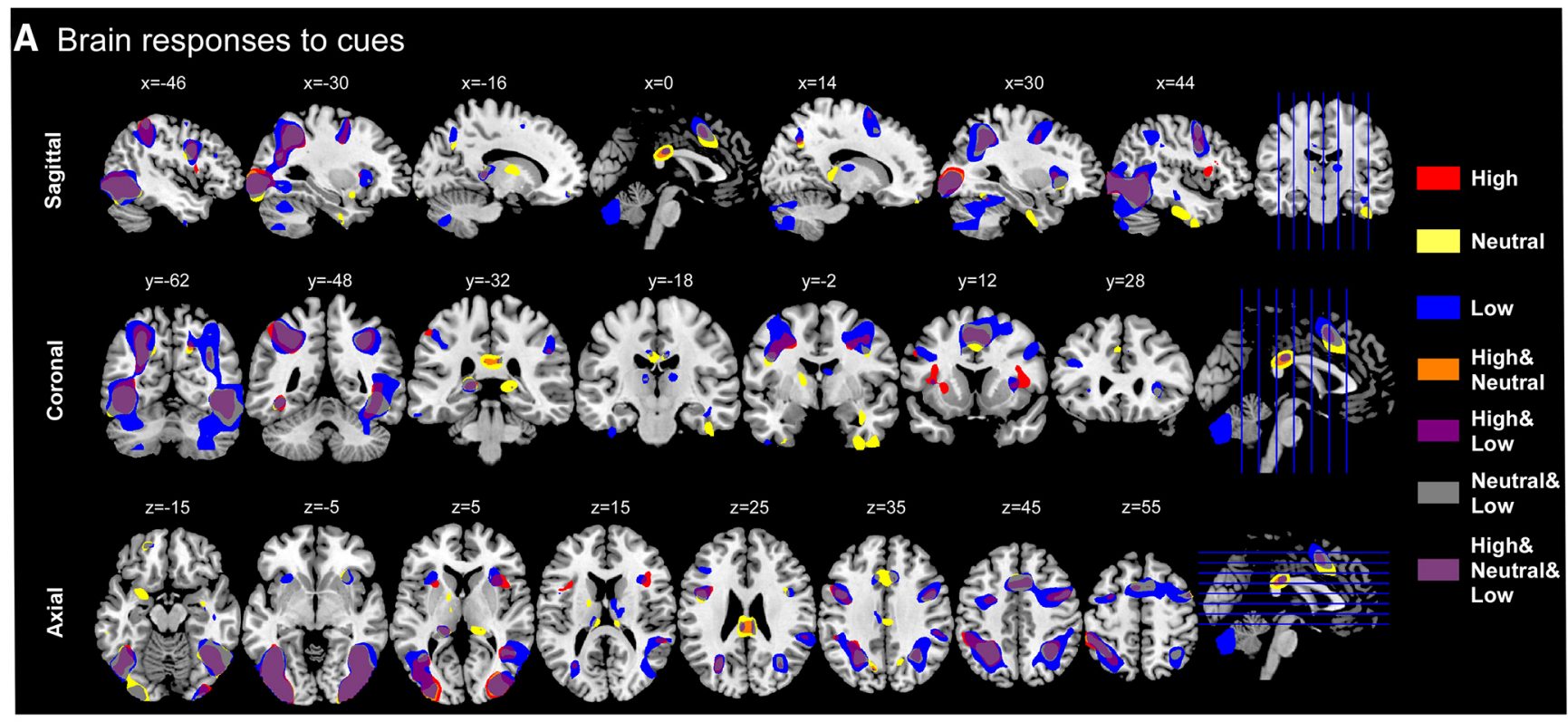

B Neutral vs. Low during anticipation

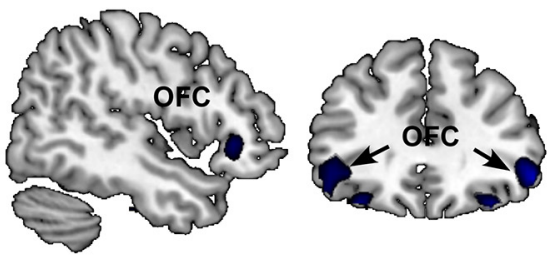

Neutral < Low

\begin{tabular}{ll} 
z score \\
-4 \\
\hline
\end{tabular}

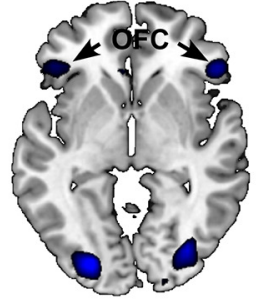

C High vs. Neutral during anticipation
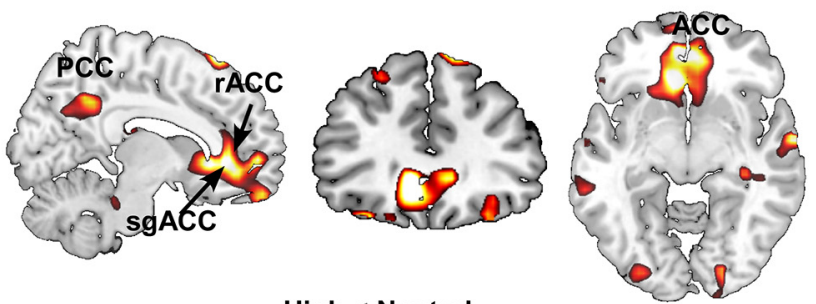

High $<$ Neutral

z score

$2 . 7 \longdiv { \text { score } } 4$

Figure 3. Brain responses associated with cue-based expectancy effects. $A$, Brain responses to low, neutral, and high cues. Visual cues elicited brain activations in a wide range of brain regions. $B$, During the pain anticipation period, positive expectation induced increased OFC activations. C, Negative expectation induced increased brain activations in the rACC and subgenual ACC (sgACC) during the pain anticipation period. All results were thresholded at $p<0.001$ at voxel level and $p_{\mathrm{FDR}}<0.05$ at cluster level. Clusters that exhibited significant differences between cues during pain anticipation are summarized in Figure 3-1 (available at https://doi.org/10.1523/JNEUROSCI.1082-19.2019.f3-1).

were calculated using the bootstrapping procedure (Hayes and Preacher, 2014). The estimate was considered statistically significant if the $95 \%$ CIs (based on 1000 bootstrap samples) excluded 0.

Multivariate pattern analysis. An MVPA was used to investigate the association between VTA-based FC and positive expectancy/negative expectancy effects at the between-subject level (Anzellotti et al., 2017; Anzellotti and Coutanche, 2018). First, multivariate FC between the VTA and each voxel in rACC (resulting in a vector pattern of VTA-rACC FC; the number of features equals to the number of voxels in rACC) and between the VTA and each voxel in left NAc (resulting in a vector pattern of VTA-NAc FC; the number of features equals to the number of voxels in NAc) were extracted for each single trial in each subject and averaged across trials for each type of cue. The differences in VTA-based multivariate FC (neutral cue vs low cue or high cue vs neutral cue) were calculated and used to predict the magnitude of conditioned pain responses. Second, the relationship between VTA-based multivariate FC (independent variables, i.e., VTA-rACC multivariate FC and VTA-NAc multivariate FC) and the magnitude of conditioned pain responses (dependent variable) was described using a multivariate linear regression model (Tu et al., 2016, 2018). To minimize the influence of different levels of pain perception following neutral cues across subjects, the percentage difference of perceived pain intensities between neutral cue and low/high cues was calculated to represent the magnitudes of conditioned pain responses for positive and negative expectations, respectively. The multivariate linear regression model was decoded using a support vector regression, as implemented in the LIBSVM toolbox (Chang and Lin,
2011), and this calculation resulted in a pattern of prediction weights for all independent variables (Tu et al., 2019b). The prediction of conditioned pain responses was achieved based on the fivefold crossvalidation. Specifically, we partitioned all subjects into five groups, four of which were used for training and the remaining one was used for testing. This procedure was repeated five times to ensure that each subject was used in the test sample once. The predicted magnitude of conditioned pain responses was calculated by taking the dot product of the pattern of prediction weights obtained from the training samples and the VTA-rACC and VTA-NAc FC from subjects in the test sample. To evaluate the prediction performance, we calculated the prediction-outcome correlation, which was defined as the correlation coefficient between the actual and predicted magnitudes of conditioned pain responses (Wager et al., 2011, 2013; Doehrmann et al., 2013; Lindquist et al., 2017).

The significance of the prediction performance was estimated using a permutation test, in which we randomly permuted the labels of the data (conditioned pain responses) before training. Cross-validation was performed on the permuted data, and the whole procedure was repeated 1000 times. If the model trained on real data labels had a predictionoutcome correlation ( $z$-scored) that exceeded the 95\% CI generated from the results of the models trained on randomly relabeled data, the prediction model was considered to be well performing.

We also examined whether BOLD signals in isolated brain regions (i.e., rACC and NAc; $\beta$ estimates at the individual level) during the pain anticipation period were predictive to conditioned pain responses. In brief, predictors consist of BOLD signals at brain voxels within rACC and 
NAc, and the relationships between the extracted BOLD signals and conditioned pain responses were modeled using the multivariate linear regression and decoded using the support vector regression.

\section{Structural MRI data analysis}

Structural MRI images were analyzed using the Computational Anatomy Toolbox (CAT12; http://www.neuro.uni-jena.de/cat/) in SPM12. Data preprocessing included bias-field and noise removal, skull stripping, segmentation into gray and white matter, and normalization to MNI space. The quality of images was assessed with the built-in image-quality rating and manually checked by authors. Gray matter was spatially smoothed using the same $5 \mathrm{~mm}$ FWHM Gaussian smoothing kernel as used in the analysis of fMRI data.

Structural properties of ventral striatum (NAc is a core part of ventral striatum) and PFC have been found to be related to dopamine-related personality traits (Schweinhardt et al., 2009). To determine whether structural properties of mesocorticolimbic pathways can predict conditioned pain responses at the between-subject level, we performed voxel-based morphometry (VBM) analysis (Ashburner and Friston, 2005) using CAT12 toolbox and voxelwise estimation of GMV for the rACC (consisting of a vector of multivariate GMV values of rACC for each subject) and left NAc (consisting of a vector of multivariate GMV values of left NAc for each subject) was extracted. Additionally, the GMV of the VTA was also extracted from each subject. The ability of the structural properties (as represented by multivariate GMV values) of these regions to predict the between-subject variability of conditioned pain responses was assessed using the same MVPA approach detailed in the previous section.

\section{Results}

\section{Behavioral results}

The perceived intensities to pain stimuli following low cue $(-)$, neutral cue $(0)$, and high cue $(+)$ were $2.69 \pm 0.18,3.32 \pm$ 0.23 , and $4.10 \pm 0.28$ (mean \pm SEM), respectively (Fig. $2 A)$. A one-way repeated-measures ANOVA showed significant differences among the three conditions $\left(F_{(2,26)}=57.6, p<0.001\right)$. Post hoc comparisons showed that perceived pain intensities were significantly higher for neutral cue than for low cue $(p<0.001)$ and significantly higher for high cue than for neutral cue $(p<0.001)$. Cue-based expectancy effects on pain did not significantly change over time, as the one-way repeated-measures ANOVA did not reveal significant differences across three sessions in the test phase for both positive $\left(F_{(2,26)}=1.71, p=0.19\right)$ and negative $\left(F_{(2,26)}=2.48, p=0.09\right)$ expectancy effects, although both effects exhibited a trend of decrease across time.

\section{Cue-based expectancy effects during pain experience and anticipation periods}

Pain stimuli elicited brain activations within a wide range of brain regions, including the bilateral insula, supplementary motor area (SMA), dorsal striatum (i.e., putamen and caudate), thalamus, primary and secondary somatosensory cortices (SI and SII), and dorsal ACC (dACC) $\left(p_{F D R}<0.05\right.$, the same hereinafter; Fig. $2 B$;
Table 1). Pain-evoked deactivations were observed in the bilateral parahippocampus (Table 1).

During the pain experience period, positive expectation induced decreased brain activations in the bilateral thalamus, bilateral insula, middle/posterior cingulate cortex (MCC/PCC), and midbrain (Fig. 2C; Table 2). In contrast, negative expectation induced increased brain activations in the bilateral insula, dACC, SMA, bilateral thalamus, bilateral putamen, MCC/PCC, bilateral dorsolateral PFC, and midbrain (Fig. 2D; Table 2), and induced decreased brain activations in the bilateral angular (Table 2). These brain regions associated with cue-based expectancy effects on pain were largely overlapping with the neurological pain signature (Wager et al., 2013). To illustrate pain-evoked brain responses following different cues in the brain regions associated with cue-based effects on pain (Atlas et al., 2010; Jensen et al., 2015; Shih et al., 2019), dACC, SMA, bilateral insula, bilateral putamen, and bilateral thalamus were selected as ROIs. Pain-evoked brain responses in these ROIs were extracted and are shown in Figure 2E.

During the pain anticipation period, different cues elicited brain activations within a wide range of overlapping brain re- 
Table 3. Clusters that exhibited significant functional connectivity with VTA during pain anticipation ${ }^{a}$

\begin{tabular}{llcrr}
\hline Area & Side & Peak $x / y / z$ & $\mathrm{~T}$ & Size \\
\hline ACC & $\mathrm{L}, \mathrm{R}$ & $9,32,25$ & 8.5 & 360 \\
$\mathrm{NAC}$ & $\mathrm{L}$ & $-16,11,2$ & 13.9 & 63 \\
$\mathrm{NAC}$ & $\mathrm{R}$ & $15,18,-5$ & 12.2 & 55 \\
$\mathrm{mPFC}$ & $\mathrm{L}, \mathrm{R}$ & $3,50,-2$ & 10.6 & 294 \\
IFG & $\mathrm{L}$ & $-40,32,14$ & 8.1 & 298 \\
IFG & $\mathrm{R}$ & $45,36,8$ & 9.2 & 223 \\
Insula & $\mathrm{L}$ & $-27,17,-11$ & 12.5 & 146 \\
Insula & $\mathrm{R}$ & $27,23,-11$ & 11.1 & 177 \\
Thalamus & $\mathrm{L}$ & $-8,-15,7$ & 11.2 & 249 \\
Thalamus & $\mathrm{R}$ & $13,-15,4$ & 11.2 & 250 \\
Caudate & $\mathrm{L}$ & $-16,14,6$ & 11.8 & 108 \\
Caudate & $\mathrm{R}$ & $14,10,4$ & 11.4 & 151 \\
Putamen & $\mathrm{L}$ & $-22,12,-2$ & 12.1 & 243 \\
Putamen & $\mathrm{R}$ & $18,8,-8$ & 10.7 & 240 \\
Hippocampus & $\mathrm{L}$ & $-17,-10,-18$ & 10.2 & 176 \\
Hippocampus & $\mathrm{R}$ & $21,-10,-20$ & 6.4 & 184 \\
Amygdala & $\mathrm{L}$ & $-19,-3,-20$ & 9.8 & 46 \\
Amygdala & $\mathrm{R}$ & $22,-3,-20$ & 10.0 & 50 \\
\hline
\end{tabular}

${ }^{a} \mathrm{FG}$, Inferior frontal gyrus.

gions (Fig. 3A). Compared with neutral cues, low cues induced increased orbitofrontal cortex (OFC) activations and decreased SMA activations (Fig. 3B; Fig. 3-1, available at https://doi.org/ 10.1523/JNEUROSCI.1082-19.2019.f3-1). In contrast, high cues induced increased brain activations in the subgenual ACC, rACC, and PCC (Fig. 3C; Fig. 3-1, available at https://doi.org/10.1523/ JNEUROSCI.1082-19.2019.f3-1).

\section{VTA-based FC during pain anticipation period}

During the pain anticipation period, VTA exhibited significant FC with a broad range of cortical and subcortical regions, including the thalamus, caudate, ACC, medial prefrontal cortex (mPFC), insula, hippocampus, amygdala, NAc, inferior frontal gyrus, and putamen (Fig. 4A; Table 3). Compared with neutral cues, the VTA showed increased FC with the rACC and NAc following low cues (Fig. 4B; Table 4). In contrast, the VTA had reduced FC with the rACC and NAc following high cues compared with neutral cues (Fig. 4C; Table 4). In the control seedbased analyses, no significant difference of SN-based FC was observed between low cues and neutral cues, as well as between high cues and neutral cues (Fig. 4D). Further, single-trial correlation analysis revealed significant negative correlations between VTA-rACC/NAc FC and perceived pain intensities (VTA-rACC: $r=-0.38 \pm 0.11, p=0.001$; VTA-NAc: $r=-0.35 \pm 0.15, p=$ 0.02 ; Fig. $4 E$ ), indicating that a stronger FC within mesocorticolimbic pathways during the pain anticipation period was associated with a lower perceived pain intensity during the pain experience period. Additionally, no significant difference in BOLD responses evoked by different cues was observed in VTA, rACC, and NAc, suggesting that the observed differences in VTA-based FC during the pain anticipation period were not likely to be contributed by differences in the magnitude of cue-evoked brain responses (Fig. 5).

VTA-based FC mediates cue-based expectancy effects on perceived pain intensity at within-subject level

Three models in the mediation analyses describing the relationships between cues, VTA-based FC, and perceived pain intensities are summarized in Figure 6. In Model 1 (Fig. 6A), we found that VTA-NAc FC significantly mediated cue-based expectancy effects on perceived pain (direct effect $=0.49, p=0.01$; indirect effect $=0.28$, 95\% CI: [0.09-0.52]). In Model 2 (Fig. 6B), we found that VTA-rACC FC significantly mediated cue-based expectancy effects on perceived pain (direct effect $=0.63, p=$ 0.002; indirect effect $=0.13,95 \% \mathrm{CI}$ : $[0.004-0.298])$. In Model 3 (Fig. 6C), two mediators (VTA-NAc FC and VTA-rACC FC) were adopted, and we found that VTA-NAc FC significantly mediated cue-based expectancy effects on perceived pain (direct effect $=0.48, p=0.02$; indirect effect $=0.26,95 \%$ CI: $[0.07-$ $0.53]$ ), while VTA-rACC FC did not (indirect effect $=0.02,95 \%$ CI: $[-0.16-0.14])$.

Pain-evoked brain responses mediate effects of VTA-based FC on perceived pain intensity at within-subject level

The model used in the structural equation modeling analysis described the mediatory role of pain-evoked brain responses in the relationship between anticipatory VTA-based FC and perceived pain intensities (Fig. 6D). The fitted model produced adequate fit indices: $\chi^{2} / \mathrm{df}=1.41$, root mean square error of approximation $=0.04$, goodness-of-fit index $=0.99$, adjusted goodness-offit index $=0.99$, comparative fit index $=0.99$, and normed fit index $=0.99$. All observed variables had high loadings $(b$ range $=$ $[0.81,0.89])$ in their respective unobserved variables. Anticipatory VTA-based FC showed a significant indirect effect $(b=$ -0.42 ; $95 \%$ CI: $[-0.83$ to -0.17$], p=0.007)$ on perceived pain intensities through pain-evoked brain responses in the thalamus and insula, but not a direct effect $(b=0.025,95 \%$ CI: $[-0.36$ to $0.44], p=0.94$ ).

\section{Functional and structural patterns in mesocorticolimbic pathways encode conditioned pain responses at between-subject level}

Functionally, we found that both VTA-rACC and VTA-NAc multivariate FCs were not able to predict positive expectationconditioned pain responses (Fig. $7 A ; r=0.13, p=0.58 ; r=0.19$, $p=0.33$; respectively) or negative expectation-conditioned pain responses (Fig. $7 B ; r=0.40, p=0.03 ; r=0.03, p=0.89$; respectively; the significance threshold was adjusted to $\left.p_{\mathrm{FDR}}<0.05\right)$ at the between-subject level. Similarly, BOLD signals at brain voxels within the rACC and NAc during the pain anticipation period were unable to predict positive or negative expectation-conditioned pain responses (positive expectation: $r=0.32, p=0.08$ for $\mathrm{rACC}$, and $r=$ $0.32, p=0.08$ for NAc; negative expectation: $r=0.20, p=0.13$ for rACC, and $r=0.41, p=0.03$ for NAc; Fig. 8).

Structurally, GMVs in the rACC and NAc were able to predict the magnitude of the positive expectation-conditioned pain responses across subjects (Fig. $7 A ; r=0.51, p=0.005 ; r=0.46, p=$ 0.01 ; respectively; the significance threshold was adjusted to $p_{\mathrm{FDR}}$ $<0.05$ ). In addition, the GMV in the rACC, but not in the NAc, was able to predict the magnitude of negative expectationconditioned pain responses across subjects (Fig. $7 B ; r=0.49, p=$ $0.007 ; r=0.14, p=0.46$; respectively). Additionally, the GMV in the VTA was able to predict the magnitude of the positive $(r=$ $0.44, p=0.02$; Fig. $8 A)$, but not the negative $(r=0.18, p=0.35$; Fig. $8 B)$, expectation-conditioned pain responses across subjects.

\section{Discussion}

In the present study, we demonstrated the relevance of mesocorticolimbic pathways in the encoding of cue-based expectancy effects on pain. At the within-subject level, the function of the mesocortical pathway (represented by the VTA-rACC FC) and the mesolimbic pathway (represented by the VTA-NAc FC) was enhanced during pain anticipation with positive expectation and 
Table 4. Clusters that exhibited significant differences of functional connectivity with VTA between cues during pain anticipation

\begin{tabular}{|c|c|c|c|c|c|c|c|c|c|}
\hline \multicolumn{5}{|c|}{ High < neutral } & \multicolumn{5}{|c|}{ Neutral $<$ low } \\
\hline Area & Side & Peak $x / y / z$ & $\mathrm{~T}$ & Size & Area & Side & Peak $x / y / z$ & $\mathrm{~T}$ & Size \\
\hline NAC & L & $-15,11,-2$ & 3.3 & 26 & NAC & L & $-1410-5$ & 5.9 & 37 \\
\hline $\mathrm{rACC}$ & $\mathrm{L}$ & $-12,38,13$ & 4.5 & 67 & rACC & L & -33519 & 4.7 & 63 \\
\hline
\end{tabular}

$A_{x=-5}$

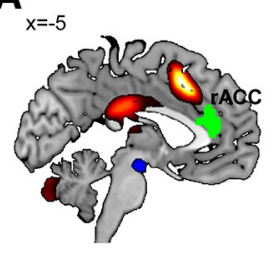

$y=-16$

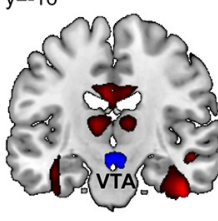

C

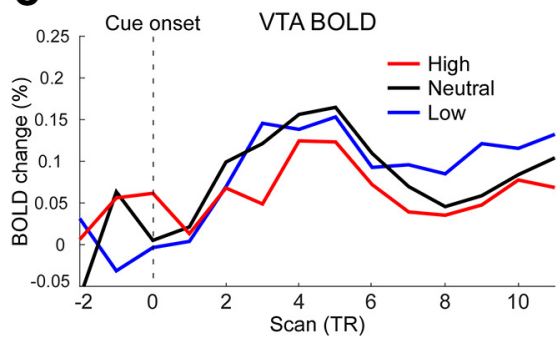

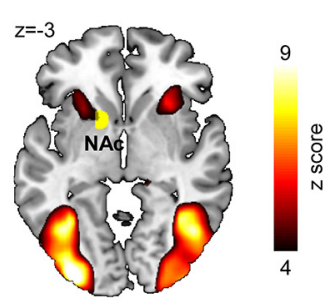

B

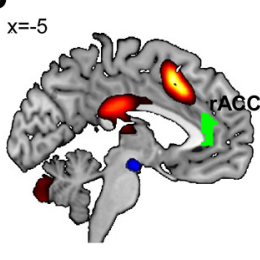

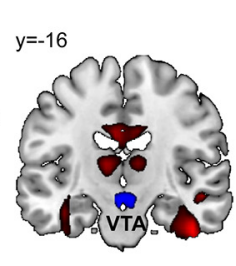

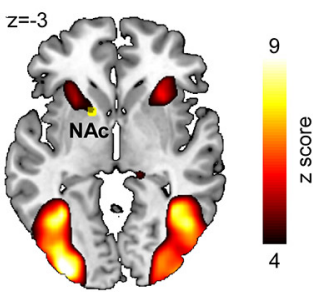

D

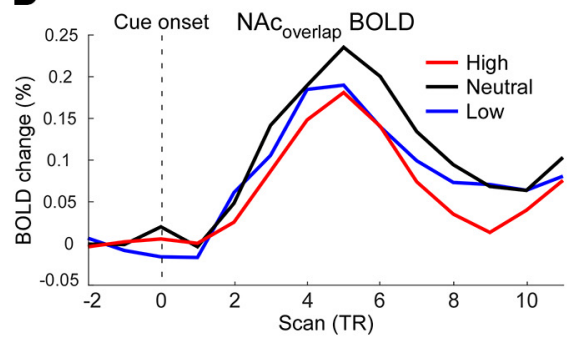

E

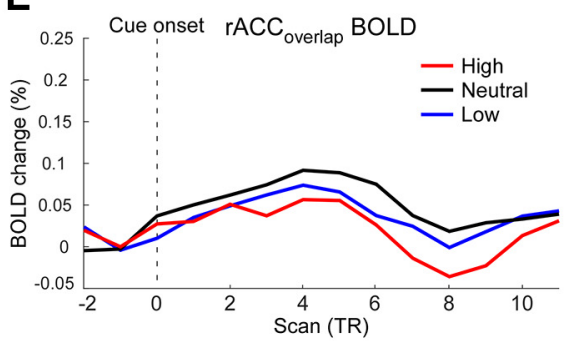

Figure 5. Cue-evoked brain responses in VTA, NAC, and rACC. $A, B$, Color-coded areas represent brain regions that were significantly activated by cues. Blue represents VTA seed. Yellow represents NAc. Green represents rACC. $A$, NAc and rACC were identified using the contrast "low versus neutral" in Figure $4 B$. $B$, NAc and rACC were identified using the contrast "high versus neutral" in Figure 4C. $C-E$, Time courses of BOLD signals following three different cues from the VTA, NAC overlap $_{\text {, and }} \mathrm{ACC}_{\text {overlap }}$ in Figure $4 E$.

inhibited during pain anticipation with negative expectation (Fig. 4B,C). Singletrial correlation analysis revealed negative relationships between FC within mesocorticolimbic pathways during the pain anticipation period and perceived pain intensities during the pain experience period (Fig. 4E). In addition, mediation analyses revealed that the cue-based expectancy effects on perceived pain intensities were mainly mediated by the VTA-NAc FC, and structural equation modeling showed that VTA-based FC influenced pain perception by modulating pain-evoked brain responses (Fig. 6). At the between-subject level, we found that FC within mesocorticolimbic pathways during the pain anticipation period was unable to predict the magnitude of conditioned pain responses (Fig. 7). In contrast, GMVs in the VTA, rACC, and NAc were able to predict the magnitude of conditioned pain responses across subjects (Figs. 7, 8). In summary, our results emphasize the important role of the mesocorticolimbic pathways underlying the cue-based expectancy effects on pain.

\section{Cue-based expectancy effects on pain}

Conditioning is known to play an important role in establishing a link between a context (cue) and the following pain stimulus, thus creating expectation for future pain responses in the same situation (Atlas and Wager, 2012). Using a
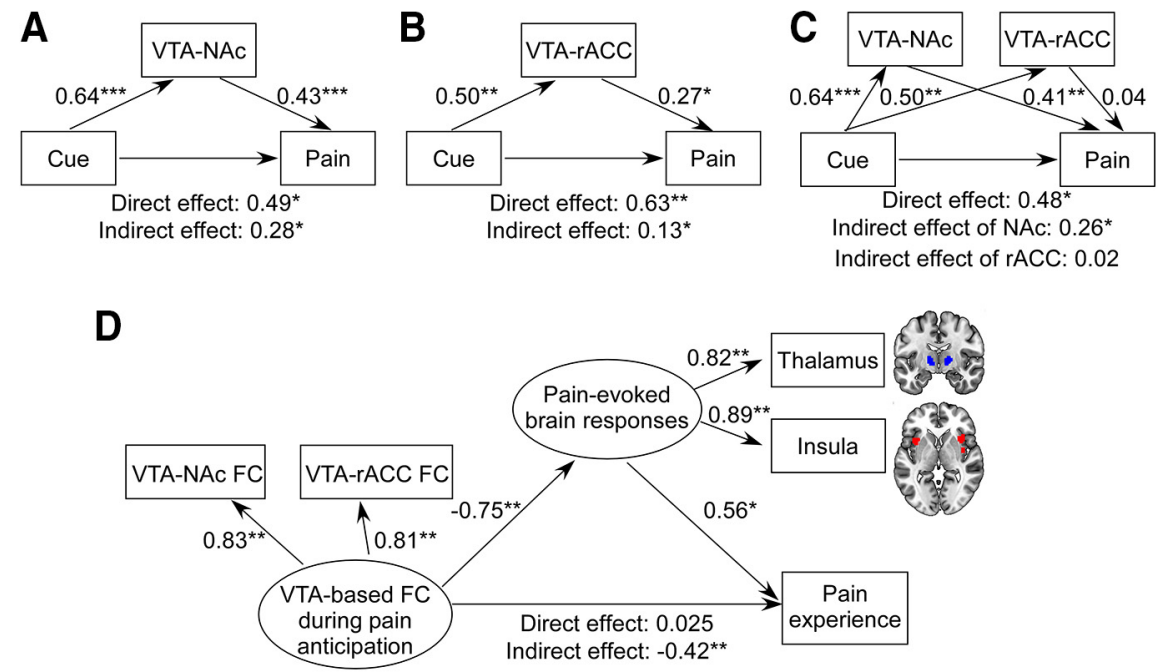

Figure 6. Path analyses of cue-based expectancy effects on perceived pain intensity. $A$, Mediation Model 1 (one mediator variable: VTA-NAC FC). Cue-based expectancy effects on perceived pain intensities were mediated by VTA-NAC FC. $\boldsymbol{B}$, Mediation Model 2 (one mediator variable: VTA-rACC FC). Cue-based expectancy effects on perceived pain intensities were mediated by VTA-rACC FC. C, Mediation Model 3 (two mediator variables: VTA-NAC FC and VTA-rACC FC). VTA-NAC FC mediated cue-based expectancy effects on perceived pain intensities, whereas VTA-rACC FC did not mediate cue-based expectancy effects on perceived pain intensities. $\boldsymbol{D}$, Structural equation modeling. VTA-based FC during pain anticipation was the predictor and loaded by VTA-NAC FC and VTA-rACCFC. Perceived pain intensity was the outcome. Pain-evoked brain responses were the mediator and loaded by the $B O L D$ responses in the bilateral thalamus and bilateral insula. VTA-based FC during pain anticipation showed a significant indirect effect on subjective pain perception through pain-evoked brain responses in the thalamus and insula, but not a direct effect. ${ }^{*} p<$ $0.05,{ }^{* *} p<0.01,{ }^{* * *} p<0.001$.

conditioning model and cue-based manipulations of stimulus expectation, previous behavioral studies have revealed that shortterm expectations that vary as a function of cue have strong effects on pain perception (Colloca et al., 2010). Further, predictive 


\section{A Positive expectancy effects}

Functional Connectivity
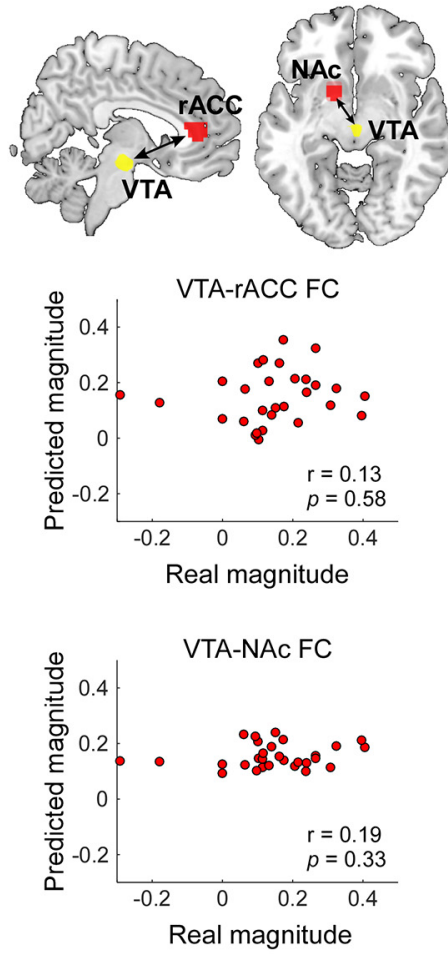

Gray Matter Volume
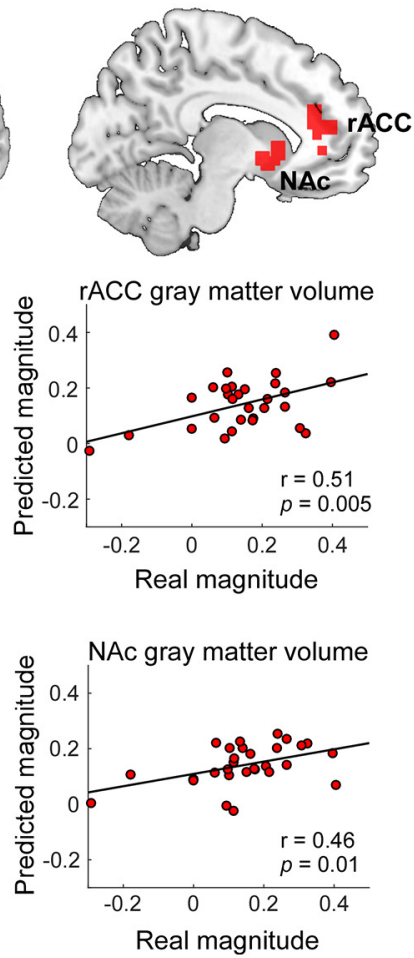

\section{B Negative expectancy effects}

Functional Connectivity
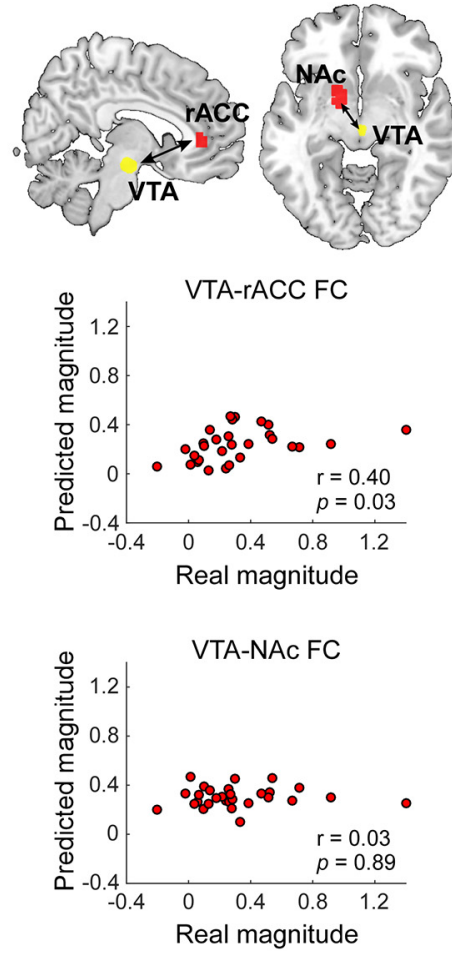

Gray Matter Volume
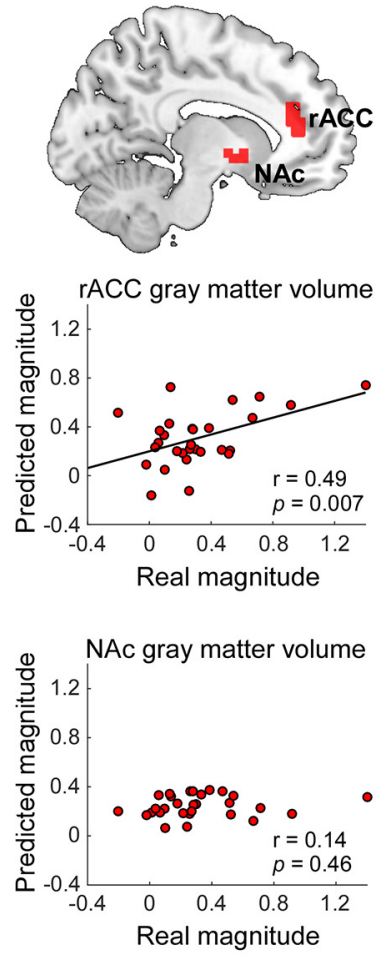

Figure 7. Brain patterns in mesocorticolimbic pathways encode individual variability of conditioned pain responses. $A$, GMVs in the rACC and NAC were able to predict the magnitude of positive expectation-conditioned pain responses across subjects. In contrast, the FC between the VTA and rACC/NAc during the pain anticipation period could not predict the magnitude of positive expectation-conditioned pain responses at the between-subject level. $\boldsymbol{B}$, The GMV of the $\mathrm{rACC}$, but not of the NAc, was able to predict the magnitude of negative expectation-conditioned pain responses across subjects. The FC between VTA and rACC/NAc could not predict the magnitude of negative expectation-conditioned pain responses at the between-subject level. FDR correction was applied $\left(p_{\mathrm{FDR}}<0.05\right)$, and the significance threshold was adjusted to $p<0.03$.

cues can be recognized subliminally, and conditioned pain responses can be elicited without conscious awareness (Jensen et al., 2012). Recent studies have extended these theories to observational conditioning, wherein subjects observe others and learn from their experience. These studies have demonstrated that social observation can also elicit expectancy effects on pain (Egorova et al., 2015; Tu et al., 2019a). In parallel, studies using fMRI have demonstrated the cue-based expectancy effects on painevoked brain responses (Koyama et al., 2005; Atlas et al., 2010; Jensen et al., 2015; Shih et al., 2019). Consistent with these studies, our results showed that cue-based expectation modulated both perceived intensity and neurobiological responses of pain (Fig. 2A-E).

Two neurotransmitters, endogenous opioid and cholecystokinin, are generally considered important in mediating the general placebo analgesia and nocebo hyperalgesia responses, respectively (Levine et al., 1978; Benedetti et al., 2006). However, they are less likely to underlie cue-based expectancy effects since expectation, unlike the effects of endogenous opioid and cholecystokinin, can vary from trial to trial. As suggested by Atlas and Wager (2012), neuromodulatory signals that mediate cuebased expectancy effects on pain are expected to be transient and reversible. Phasic dopaminergic (DA) signaling, which has been linked to the computation of prediction error and expected value, would be an appropriate candidate for mediating cue-based expectancy effects (Haruno and Kawato, 2006; Büchel et al., 2014). Indeed, a number of studies have investigated the role of DA responses in generating placebo and nocebo effects (de la Fuente-Fernández et al., 2001; Scott et al., 2007, 2008). For example, Scott et al. (2007, 2008) found that DA activity in the NAc was associated with the formation of placebo and nocebo pain responses. Results from our present study align with findings from general placebo and nocebo studies. Our study also extends previous theories by demonstrating that expectation toward pain can modulate the synchronization of key components (i.e., VTA, NAc, and rACC) in the reward circuitry. These brain regions may be associated with DA responses (Enck et al., 2008) and DA projections from tegmental to striatum and PFC (de la Fuente-Fernández et al., 2001; Lidstone et al., 2010).

\section{Neural coding of cue-based expectancy effects on within-subject variability of pain}

Previous studies have shown that expectancy effects on pain can be mediated by brain activities in localized regions during both pain anticipation and pain experience (Atlas et al., 2010; Woo et al., 2017). To investigate the role of mesocorticolimbic pathways in mediating cue-based expectancy effects, we designed a cuebased paradigm that had a long pain anticipation period (15 s, 5 times longer than previous studies) (Jensen et al., 2012, 2015). We observed that, while the VTA exhibited significant FC with several cortical and subcortical brain regions during the pain anticipation period (Fig. 4A), only the FC between the VTA and NAc and between the VTA and rACC was modulated by cuebased expectations (Fig. $4 B, C$ ). It should be noted that, while VTA-rACC FC can serve as a functional indicator of the meso- 


\section{A Positive expectancy effects} BOLD Responses

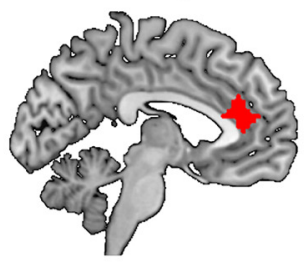

BOLD Responses

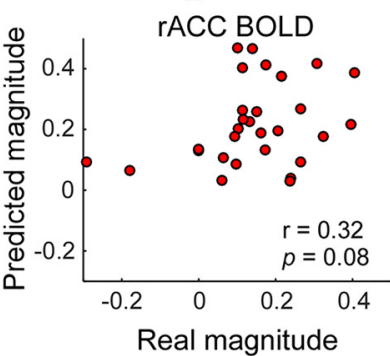

Real magnitude

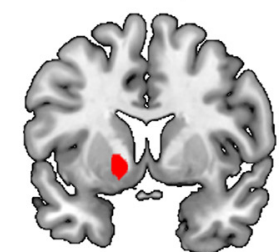

NAc BOLD

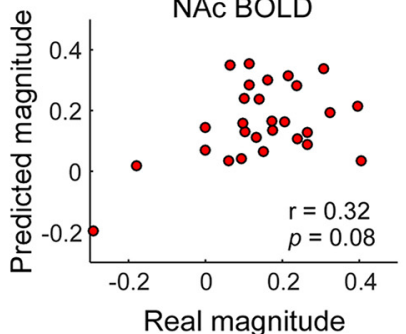

Real magnitude

\section{B Negative expectancy effects}

BOLD Responses
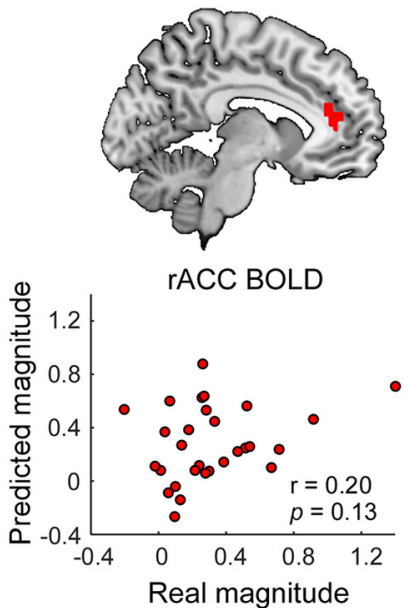

BOLD Responses

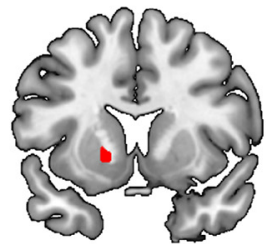

NAc BOLD

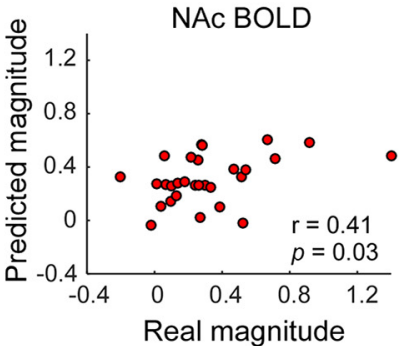

Gray Matter Volume
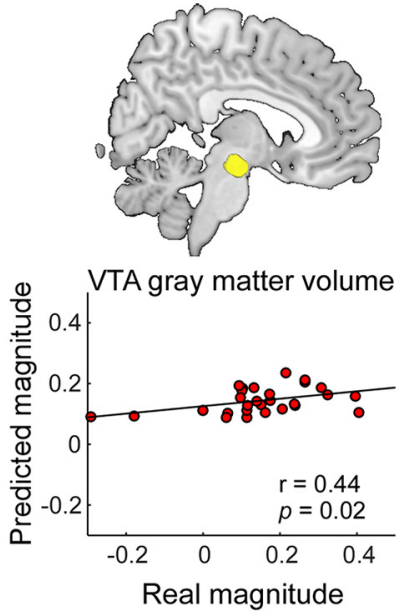

Gray Matter Volume
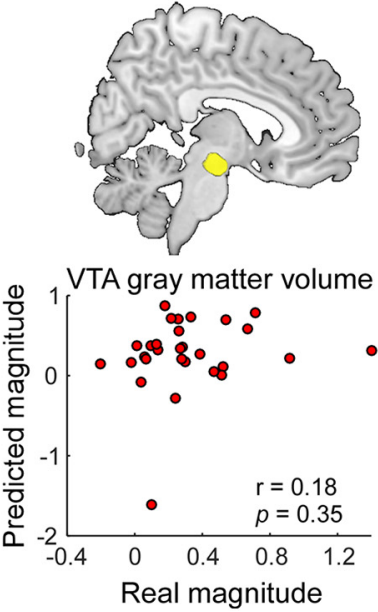

Figure 8. Prediction of individual variability of conditioned pain responses using anticipatory BOLD responses and VTA GMV. A, BOLD responses in the rACC and NAC during the pain anticipation period could not predict the magnitude of positive expectation-conditioned pain responses at the between-subject level. In contrast, the GMV in the VTA was able to predict the magnitude of positive expectation-conditioned pain responses across subjects. $B, B O L D$ responses in the rACC and NAc during the pain anticipation period, as well as VTA GMV, were not able to predict the magnitude of negative expectation-conditioned pain responses across subjects. FDR correction was applied ( $p_{\mathrm{FDR}}<0.05$ ), and the significance threshold was adjusted to $p<0.03$.

cortical pathway that is responsible for executive brain function, VTA-NAc FC is the main projection in the mesolimbic pathway related to reward and aversive processing (Gardner and Ashby, 2000). Therefore, our results indicate that both the mesolimbic and mesocortical pathways are involved in the formation of cuebased expectancy effects on pain (Ashar et al., 2017). The mediation analysis demonstrated that the effect of cue-based expectation on perceived pain was mainly mediated by VTANAc FC (Fig. $6 A-C$ ), suggesting that the mesolimbic pathway related to reward and aversive processing plays a major role in mediating cue-based expectation to relieve or enhance subsequent pain perception.

Additionally, structural equation modeling analysis showed the mediatory role of pain-evoked brain responses (in the bilateral thalamus and insula) in the relationships between anticipatory VTA-based FC and perceived pain intensities (Fig. 6D). Specifically, anticipatory VTA-based FC showed a significant indirect effect on perceived pain intensities through pain-evoked brain responses in the thalamus and insula, suggesting that anticipatory VTA-based FC modulated pain-evoked brain responses and consequently influenced pain experience. This finding is similar to that in a previous study (Atlas et al., 2010), which found that anticipatory activities in the medial OFC and ventral striatum mediated cue effects on pain-evoked brain responses in the thalamus, rostrodorsal ACC, and anterior insula, and consequently modulated pain.

Together, the mesocorticolimbic pathways may be one of the brain systems mediating expectancy effects (as indicated by the significant direct and indirect effects in Fig. $6 A-C$ ) on pain perception through pain-evoked brain responses (as indicated by the significant indirect effect but not direct effect in Fig. 6D). It is worth noting that both mediation and structural equation modeling analyses have the limitation of interpreting a causal inference between variables (e.g., there may be reciprocal causation between the mediator and the dependent variable) (MacKinnon et al., 2007), and caution should be taken when interpreting the obtained results. 


\section{Neural coding of between-subject variability of conditioned pain responses}

Since previous studies have suggested that a more efficient reward system may lead to stronger reward-related behaviors (Scott et al., 2007; Schweinhardt et al., 2009), it would be interesting to assess whether the mesocorticolimbic pathways can also encode between-subject variability of conditioned pain responses. To achieve this, we performed an MVPA to decode between-subject variability of conditioned pain responses associated with positive and negative expectations based on functional and structural features of the mesocorticolimbic pathways. We observed that functional features (i.e., VTA-rACC and VTA-NAc FC) could not significantly predict magnitudes of conditioned pain responses (Fig. 7), suggesting that functional features that varied from trial to trial may play a major role in capturing the phasic information in modulating expectancy effects on pain (e.g., expectation toward the subsequent pain stimulus). In contrast, we found that the structural features (i.e., GMVs in the NAc, rACC, and VTA) were able to predict magnitudes of conditioned pain responses across subjects. This observation is consistent with a previous study that demonstrated the role of gray matter density in the mesolimbic reward system in encoding individual differences of the placebo effect (Schweinhardt et al., 2009).

\section{Limitations and future directions}

There are several limitations of the present study that merit consideration in the future. First, the sample size was limited, especially when assessing between-subject variability of conditioned pain responses. Second, whereas the anticipatory VTA-based FC may be associated with DA responses, we were not able to measure DA responses using fMRI and did not collect DA-related psychological traits (Schweinhardt et al., 2009). Future studies with dopamine antagonists will be helpful to investigate the relationship between our findings and the DA system, and to contrast recent studies with dopamine antagonists reporting no modulation of placebo effects in the context of pain (Skyt et al., 2018; Zunhammer et al., 2018). Third, a fixed time interval during the pain anticipation period was adopted in the present study, which enabled us to make full use of data samples for a reliable estimation of the seed-based FC analyses. However, a variable time interval should be considered in the future, as it would enable a better separation of BOLD responses to successive stimuli. Fourth, the $0-10$ NRS (11 points) was used to evaluate the subjective intensity of pain perception throughout the present study, which may limit the appreciation of variability in scoring pain compared with the $0-100$ NRS (101 points).

\section{References}

Anzellotti S, Coutanche MN (2018) Beyond functional connectivity: investigating networks of multivariate representations. Trends Cogn Sci 22: $258-269$.

Anzellotti S, Caramazza A, Saxe R (2017) Multivariate pattern connectivity. PLoS Comput Biol 13:e1005799.

Ashar YK, Chang LJ, Wager TD (2017) Brain mechanisms of the placebo effect: an affective appraisal account. Annu Rev Clin Psychol 13:73-98.

Ashburner J, Friston KJ (2005) Unified segmentation. Neuroimage 26: $839-851$.

Atlas LY, Wager TD (2012) How expectations shape pain. Neurosci Lett 520:140-148.

Atlas LY, Bolger N, Lindquist MA, Wager TD (2010) Brain mediators of predictive cue effects on perceived pain. J Neurosci 30:12964-12977.

Benedetti F, Colloca L, Torre E, Lanotte M, Melcarne A, Pesare M, Bergamasco B, Lopiano L (2004) Placebo-responsive Parkinson patients show decreased activity in single neurons of subthalamic nucleus. Nat Neurosci 7:587-588.
Benedetti F, Amanzio M, Vighetti S, Asteggiano G (2006) The biochemical and neuroendocrine bases of the hyperalgesic nocebo effect. J Neurosci 26:12014-12022.

Büchel C, Geuter S, Sprenger C, Eippert F (2014) Placebo analgesia: a predictive coding perspective. Neuron 81:1223-1239.

Chang C, Lin C (2011) LIBSVM: a library for support vector machines. ACM Trans Intell Syst Technol 2:27.

Cisler JM, Bush K, Steele JS (2014) A comparison of statistical methods for detecting context-modulated functional connectivity in fMRI. Neuroimage 84:1042-1052.

Cole MW, Bassett DS, Power JD, Braver TS, Petersen SE (2014) Intrinsic and task-evoked network architectures of the human brain. Neuron 83:238-251.

Colloca L, Petrovic P, Wager TD, Ingvar M, Benedetti F (2010) How the number of learning trials affects placebo and nocebo responses. Pain 151:430-439.

Cunningham J, McCrum-Gardner E (2007) Power, effect and sample size using GPower: practical issues for researchers and members of research ethics committees. Evid Based Midwifery 5:132-136.

de la Fuente-Fernández R, Ruth TJ, Sossi V, Schulzer M, Calne DB, Stoessl AJ (2001) Expectation and dopamine release: mechanism of the placebo effect in Parkinson's disease. Science 293:1164-1166.

de la Fuente-Fernández R, Phillips AG, Zamburlini M, Sossi V, Calne DB, Ruth TJ, Stoessl AJ (2002) Dopamine release in human ventral striatum and expectation of reward. Behav Brain Res 136:359-363.

Di X, Zhang Z, Biswal BB (2018) Psychophysiological interaction and beta series correlation for task modulated connectivity: modeling considerations and their relationships. bioRxiv. 322073. Available at http://www. biorxiv.org/content/10.1101/322073v1.

Doehrmann O, Ghosh SS, Polli FE, Reynolds GO, Horn F, Keshavan A, Triantafyllou C, Saygin ZM, Whitfield-Gabrieli S, Hofmann SG, Pollack M, Gabrieli JD (2013) Predicting treatment response in social anxiety disorder from functional magnetic resonance imaging. JAMA Psychiatry 70:87-97.

Egorova N, Park J, Orr SP, Kirsch I, Gollub RL, Kong J (2015) Not seeing or feeling is still believing: conscious and non-conscious pain modulation after direct and observational learning. Sci Rep 5:16809.

Enck P, Benedetti F, Schedlowski M (2008) New insights into the placebo and nocebo responses. Neuron 59:195-206.

Fields HL (2018) How expectations influence pain. Pain 159:S3-S10.

Gardner EL, Ashby CR Jr (2000) Heterogeneity of the mesotelencephalic dopamine fibers: physiology and pharmacology. Neurosci Biobehav Rev 24:115-118.

Haruno M, Kawato M (2006) Different neural correlates of reward expectation and reward expectation error in the putamen and caudate nucleus during stimulus-action-reward association learning. J Neurophysiol 95: 948-959.

Hayes AF, Preacher KJ (2014) Statistical mediation analysis with a multicategorical independent variable. Br J Math Stat Psychol 67:451-470.

Hooper D, Coughlan J, Mullen M (2008) Structural equation modelling: guidelines for determining model fit. Electron J Bus Res Methods 6:5360.

Hu L, Bentler PM (1999) Cutoff criteria for fit indexes in covariance structure analysis: conventional criteria versus new alternatives. Struct Equ Model A Multidiscip J 6:1-55.

Hu L, Iannetti GD (2019) Neural indicators of perceptual variability of pain across species. Proc Natl Acad Sci U S A 116:1782-1791.

Jensen KB, Kaptchuk TJ, Kirsch I, Raicek J, Lindstrom KM, Berna C, Gollub RL, Ingvar M, Kong J (2012) Nonconscious activation of placebo and nocebo pain responses. Proc Natl Acad Sci U S A 109:15959-15964.

Jensen KB, Kaptchuk TJ, Chen X, Kirsch I, Ingvar M, Gollub RL, Kong J (2015) A neural mechanism for nonconscious activation of conditioned placebo and nocebo responses. Cereb Cortex 25:3903-3910.

Kline RB (2015) Principles and practice of structural equation modeling, Ed 4. New York: Guilford.

Kong J, Jensen K, Loiotile R, Cheetham A, Wey HY, Tan Y, Rosen B, Smoller JW, Kaptchuk TJ, Gollub RL (2013) Functional connectivity of the frontoparietal network predicts cognitive modulation of pain. Pain 154:459467.

Koyama T, McHaffie JG, Laurienti PJ, Coghill RC (2005) The subjective experience of pain: where expectations become reality. Proc Natl Acad Sci U S A 102:12950-12955. 
Levine JD, Gordon NC, Fields HL (1978) The mechanism of placebo analgesia. Lancet 2:654-657.

Lidstone SC, Schulzer M, Dinelle K, Mak E, Sossi V, Ruth TJ, de la FuenteFernández R, Phillips AG, Stoessl AJ (2010) Effects of expectation on placebo-induced dopamine release in Parkinson disease. Arch Gen Psychiatry 67:857-865.

Lindquist MA, Krishnan A, López-Solà M, Jepma M, Woo CW, Koban L, Roy M, Atlas LY, Schmidt L, Chang LJ, Reynolds Losin EA, Eisenbarth H, Ashar YK, Delk E, Wager TD (2017) Group-regularized individual prediction: theory and application to pain. Neuroimage 145:274-287.

MacKinnon DP, Fairchild AJ, Fritz MS (2007) Mediation analysis. Annu Rev Psychol 58:593-614.

Mouraux A, Iannetti GD, Plaghki L (2010) Low intensity intra-epidermal electrical stimulation can activate A $\delta$-nociceptors selectively. Pain 150:199-207.

Murty VP, Shermohammed M, Smith DV, Carter RM, Huettel SA, Adcock RA (2014) Resting state networks distinguish human ventral tegmental area from substantia nigra. Neuroimage 100:580-589.

Murty VP, Tompary A, Adcock RA, Davachi L (2017) Selectivity in postencoding connectivity with high-level visual cortex is associated with reward-motivated memory. J Neurosci 37:537-545.

Schweinhardt P, Seminowicz DA, Jaeger E, Duncan GH, Bushnell MC (2009) The anatomy of the mesolimbic reward system: a link between personality and the placebo analgesic response. J Neurosci 29:4882-4887.

Scott DJ, Stohler CS, Egnatuk CM, Wang H, Koeppe RA, Zubieta JK (2007) Individual differences in reward responding explain placebo-induced expectations and effects. Neuron 55:325-336.

Scott DJ, Stohler CS, Egnatuk CM, Wang H, Koeppe RA, Zubieta JK (2008) Placebo and nocebo effects are defined by opposite opioid and dopaminergic responses. Arch Gen Psychiatry 65:220-231.

Seymour B, O’Doherty JP, Koltzenburg M, Wiech K, Frackowiak R, Friston K, Dolan R (2005) Opponent appetitive-aversive neural processes underlie predictive learning of pain relief. Nat Neurosci 8:1234-1240.

Shih YW, Tsai HY, Lin FS, Lin YH, Chiang CY, Lu ZL, Tseng MT (2019) Effects of positive and negative expectations on human pain perception engage separate but interrelated and dependently regulated cerebral mechanisms. J Neurosci 39:1261-1274.

Skyt I, Moslemi K, Baastrup C, Grosen K, Benedetti F, Petersen GL, Price DD, Hall KT, Kaptchuk TJ, Svensson P, Jensen TS, Vase L (2018) Dopami- nergic tone does not influence pain levels during placebo interventions in patients with chronic neuropathic pain. Pain 159:261-272.

Tu Y, Zhang Z, Tan A, Peng W, Hung YS, Moayedi M, Iannetti GD, Hu L (2016) Alpha and gamma oscillation amplitudes synergistically predict the perception of forthcoming nociceptive stimuli. Hum Brain Mapp 37:501-514

Tu Y, Fu Z, Tan A, Huang G, Hu L, Hung YS, Zhang Z (2018) A novel and effective fMRI decoding approach based on sliced inverse regression and its application to pain prediction. Neurocomputing 273:373-384.

Tu Y, Park J, Ahlfors SP, Khan S, Egorova N, Lang C, Cao J, Kong J (2019a) A neural mechanism of direct and observational conditioning for placebo and nocebo responses. Neuroimage 184:954-963.

Tu Y, Jung M, Gollub RL, Napadow V, Gerber J, Ortiz A, Lang C, Mawla I, Shen W, Chan ST, Wasan AD, Edwards RR, Kaptchuk TJ, Rosen B, Kong J (2019b) Abnormal medial prefrontal cortex functional connectivity and its association with clinical symptoms in chronic low back pain. Pain 160:1308-1318

Tu Y, Zhang B, Cao J, Wilson G, Zhang Z, Kong J (2019c) Identifying interindividual differences in pain threshold using brain connectome: a testretest reproducible study. Neuroimage 202:116049.

Wager TD, Rilling JK, Smith EE, Sokolik A, Casey KL, Davidson RJ, Kosslyn SM, Rose RM, Cohen JD (2004) Placebo-induced changes in FMRI in the anticipation and experience of pain. Science 303:1162-1167.

Wager TD, Atlas LY, Leotti LA, Rilling JK (2011) Predicting individual differences in placebo analgesia: contributions of brain activity during anticipation and pain experience. J Neurosci 31:439-452.

Wager TD, Atlas LY, Lindquist MA, Roy M, Woo CW, Kross E (2013) An fMRI-based neurologic signature of physical pain. N Engl J Med 368:1388-1397.

Wager TD, Scott DJ, Zubieta JK (2007) Placebo effects on human muopioid activity during pain. Proc Natl Acad Sci U S A 104:11056-11061.

Wiech K, Ploner M, Tracey I (2008) Neurocognitive aspects of pain perception. Trends Cogn Sci 12:306-313.

Woo CW, Schmidt L, Krishnan A, Jepma M, Roy M, Lindquist MA, Atlas LY, Wager TD (2017) Quantifying cerebral contributions to pain beyond nociception. Nat Commun 8:14211.

Zunhammer M, Gerardi M, Bingel U (2018) The effect of dopamine on conditioned placebo analgesia in healthy individuals: a double-blind randomized trial. Psychopharmacology (Berl) 235:2587-2595. 